\title{
Variation in the fatty acid composition between and within two mantis shrimp species, Harpiosquilla harpax and Miyakea nepa: impact of season and sex
}

\begin{abstract}
Harpiosquilla harpax and Miyakea nepa are two out of 400 marine crustaceans commonly known as mantis shrimp. Mantis shrimp are commercially valuable and can be found in fish markets of some Mediterranean and many Asian countries. This study focused on a comparative analysis of variation throughout the year in regards to proximate and total fatty acid composition in the muscle of male and female $\mathrm{H}$. harpax and $\mathrm{M}$. nepa. The most abundant fatty acids identified in the muscle of both species were C16:0, C18:0, docosahexaenoic acid (DHA; C22:6n-3), eicosapentaenoic acid (EPA; C20:5n-3), and C20:4n-6. Results of this study revealed that the muscle fatty acid profiles of $\mathrm{H}$. harpax and M. nepa were not significantly different ( $p>0.05$ ), except for C14:0, C16:0, C22:0, and DHA amounts, and were mostly influenced by sex within each sampling month. Generally, the muscle of $\mathrm{H}$. harpax contained higher $(\mathrm{p}<0.05)$ levels of DHA than the muscle of $\mathrm{M}$. nepa. The male mantis shrimp, within each species and most of the sampling months, appeared to have higher ( $\mathrm{p}<0.05)$ DHA, total $n-3$ polyunsaturated fatty acid (PUFA), and PUFA/saturated fatty acid ratio compared to the females. Both species could be considered as a good source of n-3 PUFA in the human diet.
\end{abstract}

Keyword: Fatty acid; Mantis shrimp; Season; Sex; Harpiosquilla harpax; Miyakea nepa 